\title{
NORMAL-CONDUCTING SEPARATION AND COMPENSATION DIPOLES FOR THE LHC EXPERIMENTAL INSERTIONS
}

\author{
V. Petrov, D. Gurov, O. Kiselev, I. Morozov, A. Ogurtsov, E. Ruvinsky, A. Sukhanov, K. Zhilayev, \\ The Budker Institute of Nuclear Physics, BINP, 630090, Novosibirsk, Russia \\ S. Bidon, D. Cornuet, D. Gérard, O. Hans, W. Kalbreier, S. Ramberger, G. de Rijk, CERN, \\ CH-1211, Geneva 23, Switzerland
}

\begin{abstract}
The experimental insertions of the LHC make use of normal-conducting magnets to provide for part of the beam separation and to compensate the effect of two large spectrometer dipoles. Three different types with respect to the length were designed and are based on the same type of lamination. The main type of magnet MBXW has a core length of $3.4 \mathrm{~m}$ while the MBXWT and MBXWS magnets are $1.5 \mathrm{~m}$ and $0.75 \mathrm{~m}$ long versions respectively. The magnet design was done in collaboration between CERN and BINP and the dipole magnets are produced by BINP. So far all three MBXWS magnets, all three MBXWT magnets and fifteen of twenty-nine MBXW magnets have been manufactured and delivered to CERN. The report presents the main design issues and results of the acceptance tests including mechanical, electrical and magnetic field measurements.
\end{abstract}

\section{INTRODUCTION}

The experimental insertions of the LHC make use of normal-conducting magnets to provide for part of the beam separation and to compensate the effect of two large spectrometer dipoles [1]. In the interaction regions IR1 for the ATLAS experiment and IR5 for the CMS experiment, each of the optical elements D1 for beam separation on either side of the experiment consists of 6 MBXW dipoles. Each magnet has a core length of $3.4 \mathrm{~m}$, a large single aperture with a gap height of $63 \mathrm{~mm}$. The magnets are integrated into the optical lattice of the LHC, so a high quality magnetic field must be provided over the entire energy range from $0.45 \mathrm{TeV}$ to $7.5 \mathrm{TeV}$. This corresponds to a magnetic field ranging from $0.08 \mathrm{~T}$ to $1.38 \mathrm{~T}$ in the MBXW magnets, with an ultimate field of 1.48T. The MBXWT and MBXWS magnets are shorter versions of the MBXW magnet and will be used as vertical and horizontal compensation dipoles for the spectrometer dipoles in IR2 for the ALICE and in IR8 for the LHCb experiments respectively. Additionally one MBXW magnet serves as a main compensator for the LHCb experiment.

The report presents the main design issues and results of the acceptance tests including mechanical, electrical and magnetic field measurements.

\section{MAGNET DESIGN AND OPTIMIZATION}

The magnet was designed through collaboration between CERN and BINP. One of the main issues in the design was the requirement for the low saturation of the magnetic steel and the size restrictions in order to fit the magnets within the existing LHC tunnels. The numerical design of the insertion magnets was done with the help of the electro-magnetic field computation code MERMAID [2] developed at BINP. This FEM code offers 2D and 3D simulations. The magnet features an H-type shape as this provides the most homogenous field at minimal transversal dimensions (Fig. 1).

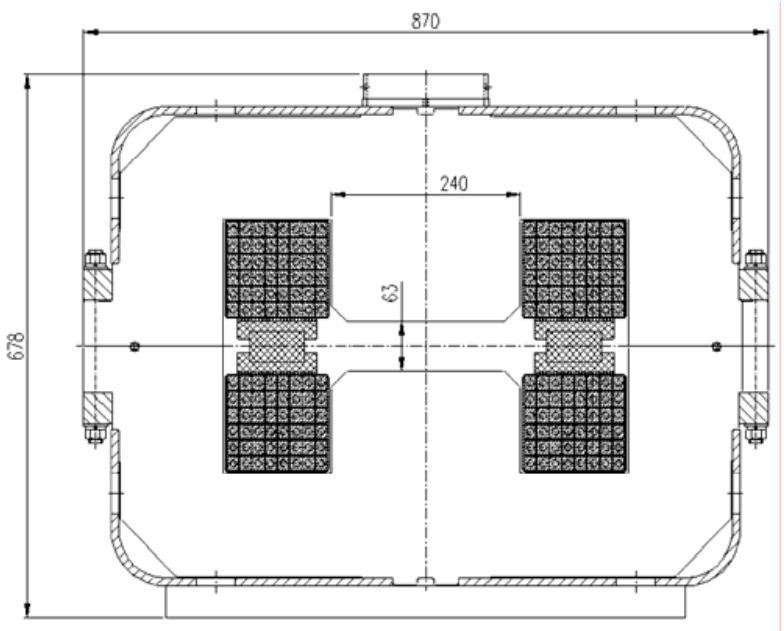

Figure 1: Cross-section of the MBXW magnet.

The pole distance measures $63 \mathrm{~mm}$. In order to achieve a good field region of $\pm 41 \mathrm{~mm}$ width with a high field

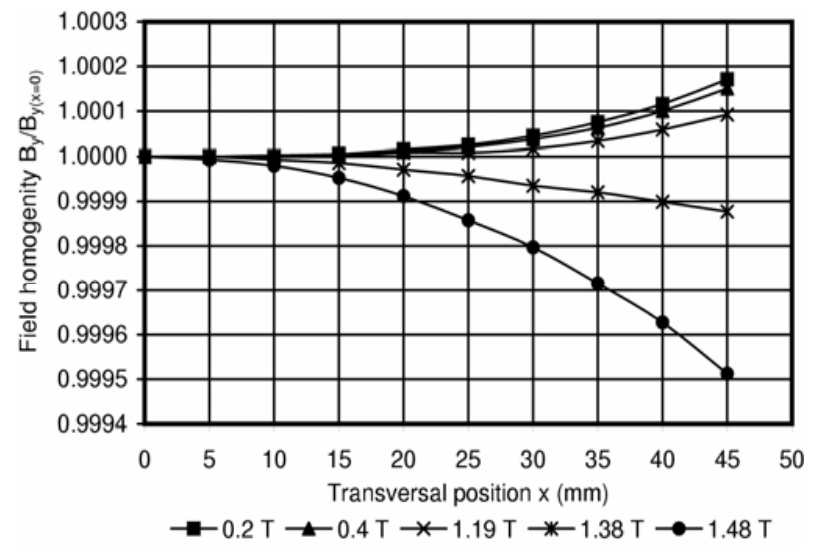

Figure 2: Field homogeneity of the magnetic flux density at different field levels (2D calculation).

quality, the pole width of $240 \mathrm{~mm}$ has been chosen. The cross-section of the magnet yoke has been defined with 
respect to the pole width and in order to limit saturation effects in the yoke.

The shape of the pole shims in principle should be different for small and large operating fields (Fig. 2). To achieve the required operating range, it has been optimized with an objective function covering various field levels. Requirements for the precision of the pole profile are determined by the tolerances for the field homogeneity.

The modeling of the magnet end profile was carried out in two stages. First, the shape of the chamfer with a slant of 45 degrees has been optimized over the whole operation range by 2D modeling such as to minimize the deviation of the effective length of the magnet from the geometrical length of the yoke. Even though the length of the magnet is much larger than the pole gap, the pole end shape still had to be corrected to limit field errors due to stray fields. In order to guarantee the required field homogeneity, the shape of the end chamfer was corrected by 3D modeling, (Fig. 3-4).

\section{MBXW MECHANICAL DESIGN}

The main requirements to the MBXW, MBXWT and MBXWS magnets are presented in Table 1. The main principles of the MBXW, MBXWT and MBXWS magnets are similar to the MBW magnets [3]. The dipoles are assembled from two half-cores joined together by tension plates. Such a structure allows opening the magnet without welding after delivery to CERN. The half-cores are made of precision-punched CockerillUsinor Magnetil 15D4 steel laminations of $1.5 \mathrm{~mm}$ thickness, assembled together with $80 \mathrm{~mm}$ thick end plates and $10 \mathrm{~mm}$ thick angular plates along the halfcores. The end plates are made from solid steel.

In order to improve the uniformity of magnetic features of the stacks, the laminations are shuffled before the stacking. The shuffling procedure is determined in order to compensate both the thickness variation over the steel sheet and the difference in magnetic features of

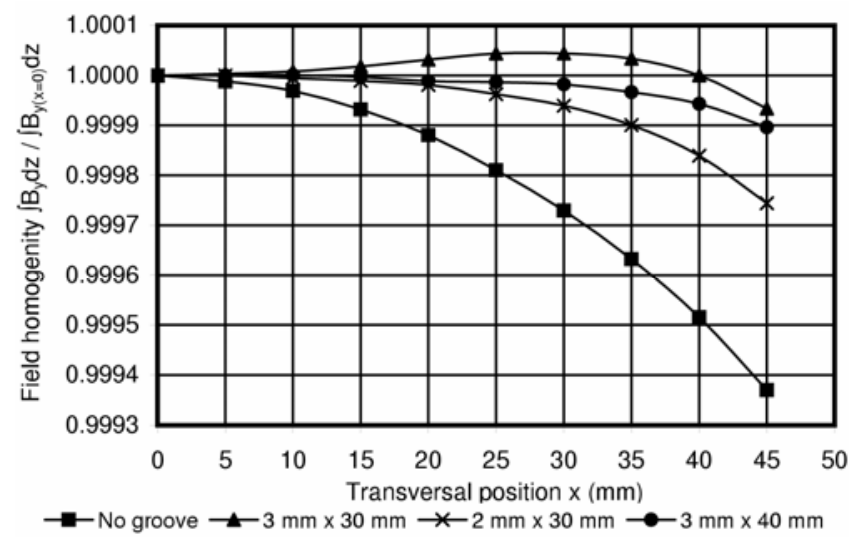

Figure 3: MBXW integral field after correction by a groove of different dimensions in the end chamfer at $\mathrm{B}=1.38 \mathrm{~T}$ (3D calculation). thepackages of steel. In the process of stacking, groups of 27 laminations are alternately rotated relative to the

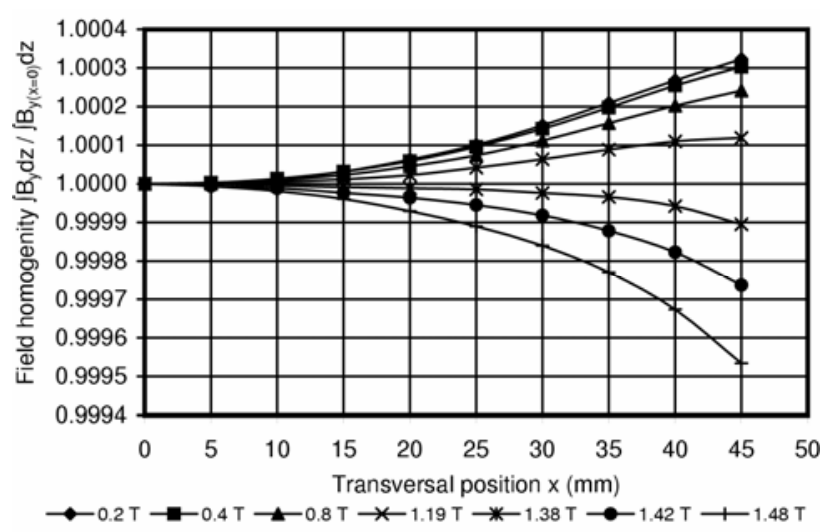

Figure 4: Field homogeneity of the integral magnetic flux density at different field levels after correction of the end chamfers (3D calculation, the groove is $3 \mathrm{~mm} \times 40 \mathrm{~mm}$ ).

vertical axis. The laminations are uniformly distributed along the stack by compressing them with a tightening force of $100 \mathrm{kN}$ applied every $0.5 \mathrm{~m}$ in the process of stacking. After stacking and clamping, the stack is inspected to make sure that the mating surfaces of all laminations are in contact with the reference surfaces of the fixture.

Table 1: Main design parameters of the magnets

\begin{tabular}{|l|c|c|c|c|}
\hline & & MBXW & MBXWT & MBXWS \\
\hline Number of magnets & & 29 & 3 & 3 \\
\hline Mag. field orientation & & vertical & horizont. & vertical \\
\hline Gap height & $\mathrm{mm}$ & 63 & 63 & 63 \\
\hline Injection field level & $\mathrm{T}$ & 0.083 & 0.076 & 0.088 \\
\hline Nominal field level & $\mathrm{T}$ & 1.29 & 1.17 & 1.37 \\
\hline Ultimate flux density & $\mathrm{T}$ & 1.48 & 1.28 & 1.41 \\
\hline Field quality @ nom. & & $\leq \pm 2 \cdot 10^{-4}$ & $\leq \pm 1 \cdot 10^{-3}$ & $\leq \pm 1 \cdot 10^{-3}$ \\
\hline Good field region & $\mathrm{mm}$ & \pm 41 & \pm 17 & \pm 17 \\
\hline Nominal current & $\mathrm{A}$ & $\sim 700$ & $\sim 600$ & $\sim 730$ \\
\hline Ultimate current & $\mathrm{A}$ & 830 & 650 & 800 \\
\hline Magnetic length & $\mathrm{m}$ & 3.4 & 1.53 & 0.78 \\
\hline End chamfers & $\mathrm{mm}$ & 43.5 & 15 & 15 \\
\hline Cooling circuits & & 6 & 2 & 2 \\
\hline Total mass & $\mathrm{kg}$ & 11500 & 5800 & 3000 \\
\hline Integral field disp. & & \multicolumn{5}{|c|}{$\leq \pm 3 \cdot 10^{-3}$} \\
\hline Overall width & $\mathrm{mm}$ & \multicolumn{4}{|c|}{2 coils $\times 48$ turns } \\
\hline Coils & & $\mathrm{mm}$ & \multicolumn{5}{|c|}{$18 \times 15$} \\
\hline Conductor $\mathrm{h} \times \mathrm{w}$ & $\mathrm{m}$ & \\
\hline
\end{tabular}

The stacking factor value must be $\geq 98 \%$ and its variation must be kept within $\pm 0.2 \%$.

Additionally to the control of the laminations, the magnet yokes are checked geometrically at BINP and after delivery to CERN. In order to reach the required 
field quality, mechanical tolerances are tight. In particular the planarity and the twist of the MBXW magnets are verified at the access holes to stay within $\pm 0.2 \mathrm{~mm}$ and 1 mrad respectively.

Each magnet has two excitation coils and each coil consists of three pancakes. Each pancake consists of 2 layers of 8 turns of a hollow copper conductor $(18 \mathrm{~mm} \times 15 \mathrm{~mm}$ with a bore of $8 \mathrm{~mm}$ in diameter for water cooling). Each pancake is wound and wrapped separately with a glass fiber tape for insulation. Then three pancakes are put together and a ground insulation layer is applied with a thicker tape. The complete coil is vacuum impregnated with radiation resistant epoxy resin. The impregnating compound is composed of 100 parts of epoxy resin ED-16, 37 parts of maleic anhydride (MA), 20 parts of polyester plasticizer MGF- 9 and 0.5 parts of accelerator triethanolamine (TEA). These materials are fully mixed, followed by further thorough mixing during degassing. The resin is vacuum degassed (below $1 \mathrm{mbar}$ at a temperature of $65-70{ }^{\circ} \mathrm{C}$ ) until the mixture is free from air and impurities with low boiling points. For degassing, the mould with the assembled coils is heated up to $65-70{ }^{\circ} \mathrm{C}$ and evacuated to below 1 mbar during several hours. The coils are then fully impregnated with the resin compound. During the impregnation process the temperature of the resin and the mould are maintained at a constant temperature.

In the course of manufacturing the prototype coils were examined by thermal cycling with a heat-up to $90^{\circ} \mathrm{C}$ and subsequent cool-down to $30^{\circ} \mathrm{C}$. The cycle duration is 20 to 30 minutes. A coil is considered to be good if it stands 25 high voltage cycles and subsequent tests of the interturn insulation, and if the insulation shows no cracks, voids, shells, or de-lamination of the insulation from the conductor.

\section{MAGNETIC MEASUREMENTS}

Magnetic measurements of all the dipoles are done by BINP using a Hall probe array of 19 Hall probes located on a common plate. The centers of the Hall probes are situated in the horizontal plane, with a maximum spacing of $5.0 \mathrm{~mm}$ (the spacing was measured with 5-7 $\mu \mathrm{m}$ accuracy), transverse to the beam direction, and they provide a region of measurement of $\pm 45 \mathrm{~mm}$. The Hall probe array is moved along the magnetic axis by means of a drive mechanism including a stepping motor, reducer and high accuracy $1.2 \mathrm{~m}$ long screw. The probe positioning along the axis is precise within $0.1 \mathrm{~mm}$. The Hall probes are calibrated vs. NMR probes in a special precise calibration magnet at all the levels of measured fields. The absolute error of each individual device is below $\pm 5 \cdot 10^{-5} \mathrm{~T}$ after applying several correction methods. In the course of measurement the field map was measured along the magnet in the region of $\pm 2 \mathrm{~m}$ relative to the center, in the current range from $100 \mathrm{~A}$ to $830 \mathrm{~A}$. The measurement step along the beam axis is $2.5 \mathrm{~cm}$ in the central part of the magnet and $1 \mathrm{~cm}$ in the stray field region. Before starting the magnetic measurement, each magnet undergoes at least 4 current cycles, from 0 to $835 \mathrm{~A}$, at a ramp rate of less than $50 \mathrm{~A} / \mathrm{s}$, and a flat-top stabilization for more than $20 \mathrm{~s}$ at the maximum value. Current regulation always goes from lower to higher current values. The overshoot at the end of every current sweep is kept below $0.1 \%$. The current stability during the measurement is controlled with a DCCT supplied by CERN in order to reach a precision of measurement within the group of MBXW magnets of $\pm 3 \cdot 10^{-4}$ [4].

After preliminary measurements, without correction of the end chamfers, the relevant shimming was performed. Fig. 5 shows comparative results of magnetic field integral measurements before and after correction for the MBXW magnet. Some series magnets have also been measured magnetically at CERN using a rotating coil setup. Both methods give comparable results and show a good agreement of the measurement results with the design values.

\section{CONCLUSION}

The production of the MBXW type magnets at BINP is well advanced. Magnetic measurement data together with an inspection report and reception tests establish the quality assurance record for the acceptance of the magnets at CERN. The results show that the design

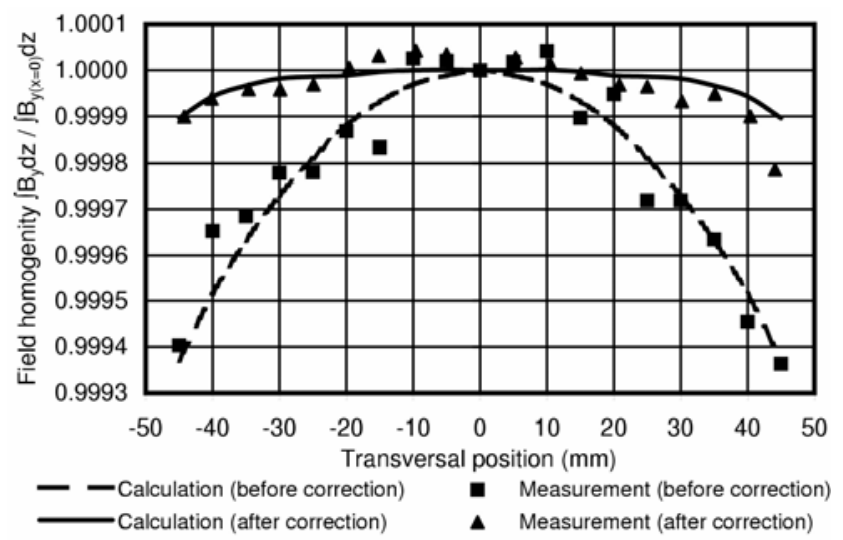

Figure 5: MBXW integral field homogeneity before and after correction at the end chamfers at $\mathrm{B}=1.38 \mathrm{~T}$.

fulfills the specification requirements and confirms the production methods at BINP. At the moment all twentynine MBXW magnets, three MBXWT magnets and three MBXWS magnets have been manufactured and delivered to CERN.

\section{REFERENCES}

[1] LHC Design Report, Vol. 1, The LHC Main Ring, CERN2004-003-V1, CERN, Geneva, 2004

[2] MERMAID User Guide, Novosibirsk, 1994

[3] S.Bidon et al. "Normal Conducting Separation Dipoles for the LHC Beam Cleaning Insertions", IEEE Trans. Appl. Supercond., vol. 14, no. 2, pp.437-440, June 2004. 